\title{
Orbital Evolution and Orbital Phase Resolved Spectroscopy of the HMXB Pulsar 4U 1538-52 with RXTE-PCA and BeppoSAX
}

\author{
U. Mukherjee ${ }^{1, *}$, H. Raichur ${ }^{1,2}$, B. Paul ${ }^{1}$, S. Naik ${ }^{3} \&$ N. Bhatt ${ }^{4}$ \\ ${ }^{1}$ Tata Institute of Fundamental Research, Homi Bhabha Road, Colaba, Mumbai 400 005, India. \\ *e-mail: uddipan@mailhost.tifr.res.in \\ ${ }^{2}$ Joint Astronomy Program, Indian Institute of Science, Bangalore 560 012, India. \\ ${ }^{3}$ Institute of Space and Astronautical Science, 3-1-1 Yoshinodai, Sagamihara, Kanagawa 229 \\ 8510, Japan. \\ ${ }^{4}$ Nuclear Research Laboratory, Bhabha Atomic Research Centre, Mumbai 400 085, India.
}

Received 2006 January 23; accepted 2006 August 8

\begin{abstract}
We report here results from detailed timing and spectral studies of the high mass X-ray binary pulsar 4U 1538-52 over several binary periods using observations made with the Rossi X-ray Timing Explorer (RXTE) and BeppoSAX satellites. Pulse timing analysis with the 2003 RXTE data over two binary orbits confirms an eccentric orbit of the system. Combining the orbitial parameters determined from this observation with the earlier measurements we did not find any evidence of orbital decay in this X-ray binary. We have carried out orbital phase resolved spectroscopy to measure changes in the spectral parameters with orbital phase, particularly the absorption column density and the iron line flux. The RXTE-PCA spectra in the 3-20 keV energy range were fitted with a power law and a high energy cut-off alongwith a Gaussian line at $\sim 6.4 \mathrm{keV}$, whereas the BeppoSAX spectra needed only a power law and Gaussian emission line at $\sim 6.4 \mathrm{keV}$ in the restricted energy range of $0.3-10.0 \mathrm{keV}$. An absorption along the line of sight was included for both the RXTE and BeppoSAX data. The variation of the free spectral parameters over the binary orbit was investigated and we found that the variation of the column density of absorbing material in the line of sight with orbital phase is in reasonable agreement with a simple model of a spherically symmetric stellar wind from the companion star.
\end{abstract}

Key words. Stars: binaries: eclipsing-stars: pulsars: individual: 4U 1538-52-X-rays: stars.

\section{Introduction}

The orbits of binary systems evolve with time due to orbital angular momentum changes. This change of orbital angular momentum can happen due to mass loss from the system, mass transfer within the system and tidal interactions between the components. X-ray binary pulsars are suitable candidates to study such orbital evolution because of the following reasons. Firstly, in all these systems the normal companion 
star is transferring matter to the compact star either through wind capture or through Roche-lobe overflow. Secondly, the strong gravitational pull of the compact object raises tides on the companion star which tend to circularize and synchronize the binary orbit. This causes orbital angular momentum changes which result in the change of orbital period. Thirdly, pulse timing analysis allows us to obtain an orbital ephemeris history with an accuracy upto a few seconds. Thus monitoring X-ray binary pulsars over a time span of few decades allows us to measure the rate of change of the orbital period. For most X-ray binaries the orbital ephemeris clearly deviates from the linear trend. Including a quadratic term improves the fit in several cases (Cen X-3, Nagase et al. 1992; LMC X-4, Levine et al. 2000, Naik \& Paul 2004; SMC X-1, Wojdowski et al. 1998) indicating a non-vanishing orbital period derivative. The many X-ray satellite missions have thus made it possible to measure the rate of change of the orbital period, showing that for High Mass X-ray Binaries (HMXB) the orbital evolution time scales are of the order of a million years.

The X-rays emitted from the compact object in HMXBs and subsequently reprocessed in the stellar wind can be used to probe the stellar wind of the massive companion, a supergiant or a Be star. An investigation of the X-ray spectrum from the compact object at different orbital phases can give useful insight into the morphology of the stellar wind. The absorbing column density in the line of sight would vary with orbital phase and is a function of the mass-loss rate, stellar wind velocity and acceleration method and also depends on any anisotropy in the stellar wind structure. Moreover, a binary system with an elliptical orbit would be a better candidate in this type of study since it would have a larger variation of the parameters that determine the column density at different orbital phases. If the compact object is a pulsar, the orbit can often be determined precisely, which is an added advantage. Orbital phase resolved spectroscopy was carried out recently on some HMXB pulsars like X $1908+075$ (Levine et al. 2004) and GX 301-2 (Mukherjee \& Paul 2004) and very different results were obtained.

$4 \mathrm{U} 1538-52$ is an X-ray pulsar, first detected with the UHURU satellite (Giacconi et al. 1974). Regular X-ray pulsations with a period of $529 \mathrm{~s}$ were later discovered with Ariel 5 and OSO- 8 observations by Davison et al. (1977). In addition, the OSO-8 observations revealed a clear orbital modulation of the pulsation period and also showed good evidence for an eclipse lasting 0.6 day recurring with a period of 3.75 days. The optical counterpart was found to be an early B type supergiant star with $\mathrm{H}$-alpha emission lines (Parkes et al. 1978). Features in the optical spectrum suggested a distance to the source of $\sim 5.5 \mathrm{kpc}$ and the mass-loss rate for the companion was estimated to be $\sim 10^{-6} \mathrm{M}_{\odot} \mathrm{yr}^{-1}$.

Makishima et al. (1987) using TENMA and Robba et al. (1992) using EXOSAT observed pulse periods of $\sim 530$ s with an average spin-down rate of $\dot{P} \sim 3.9 \times$ $10^{-9} \mathrm{~s} \mathrm{~s}^{-1}$. Subsequently, the Burst and Transient Source Experiment (BATSE) onboard the Compton Gamma Ray Observatory (CGRO) detected a reversal of the long term spin down to spin up, probably in 1988 (Rubin et al. 1997), which has also been confirmed by BeppoSAX (Robba et al. 2001). The spectrum has an iron $\mathrm{K}$ fluorescence line at $6.4 \mathrm{keV}$, a cyclotron absorption line near $20 \mathrm{keV}$ and is well fitted with a power law and high energy cut-off at $\sim 16 \mathrm{keV}$ (Clark et al. 1990; Robba et al. 2001). X-ray eclipse phenomena were investigated by Clark et al. (1994) with the GINGA data. 
$4 \mathrm{U} 1538-52$, a wind fed HMXB pulsar with a compact eccentric orbit $(e \sim 0.18)$ is very suitable for the study of the orbital evolution and wind structure of the companion star. In this paper, we report pulse timing measurements with a new observation with the RXTE and also the out-of-eclipse spectral evolution with orbital phase. Better spectral coverage with BeppoSAX (going down to $0.3 \mathrm{keV}$ ) enables us to constrain the column density values well. We consider the pulsar to be immersed in a spherically symmetric stellar wind from the companion star and compare model column density profiles with the observed profile.

\section{Observations}

We observed the source with the Proportional Counter Array (PCA) of RXTE from 2003-07-31 to 2003-08-07 covering the out of eclipse phases for two binary orbits. There were twenty-five observations, each corresponding to one orbit of the satellite. Each observation was of 1.5-6.0 ks duration. For spectral analysis, we also used an archival observation from RXTE spanning from 1997-01-01 to 1997-01-05, having fourteen satellite orbit data. Each observation had 1.6-10.0 ks of on-source time. On an average, three PCUs (see next paragraph) were ON for the observations in 2003 while all the five PCUs were ON for the observations made in 1997. Both these datasets from RXTE provide a comprehensive orbital coverage. We have also used archival BeppoSAX data obtained between 1998-07-29 to 1998-08-01, covering one binary orbit. There were forty segments in this observation; one for each satellite orbit, with an exposure of $1.7-4.0 \mathrm{ks}$. The motivation for using the BeppoSAX observations is to have a better spectral coverage since the lower values of column densities are measured more accurately with BeppoSAX, which has a good response at low energies.

RXTE consists of a large-area proportional counter array (PCA), with five Xenon proportional counter units (PCUs) and sensitive in the energy range of 2-60 keV with an effective area of $6250 \mathrm{~cm}^{2}$ (Jahoda et al. 1996). It also consists of a high energy crystal scintillation experiment (HEXTE; 15-200 keV; $1600 \mathrm{~cm}^{2}$ area), and a continuously scanning all-sky monitor (ASM; $2-10 \mathrm{keV} ; 90 \mathrm{~cm}^{2}$ ). The RXTE-ASM long term light curve of $4 \mathrm{U} 1538-52$ folded at the orbital period is shown in Fig. 1 which clearly shows the eclipse with a slow ingress and a sharp egress. The BeppoSAX observatory has a set of Narrow Field Instruments (NFI) comprising one Low Energy Concentrator Spectrometer (LECS; $0.1-10 \mathrm{keV}, 22 \mathrm{~cm}^{2} @ 0.28 \mathrm{keV}$ ), three Medium Energy Concentrator Spectrometers (MECS; $1.3-10 \mathrm{keV}, 150 \mathrm{~cm}^{2} @ 6 \mathrm{keV}$ ), one High Pressure Gas Scintillator Proportional Counter (HPGSPC; 4-120 keV, $240 \mathrm{~cm}^{2} @ 30 \mathrm{keV}$ ), and a Phoswich Detection System (PDS; 15-300 keV, $\left.600 \mathrm{~cm}^{2} @ 80 \mathrm{keV}\right)$. A detailed description of BeppoSAX can be found in Boella et al. (1997).

\section{Timing analysis and results}

Only the 2003 RXTE-PCA data were used for a timing analysis. The light curve was extracted from event mode data with time resolution of $0.125 \mathrm{~s}$. Only photons detected in the top two layers were used. The photon arrival times were then corrected to the solar system barycenter. Figure 2 shows the complete barycentered light curve for the August 2003 observation with RXTE-PCA.

The light curve shows variability at time scales other than the regular $526.85 \mathrm{~s}$ pulses from the neutron star. The pulsed emission is strong enough for individual pulses to 


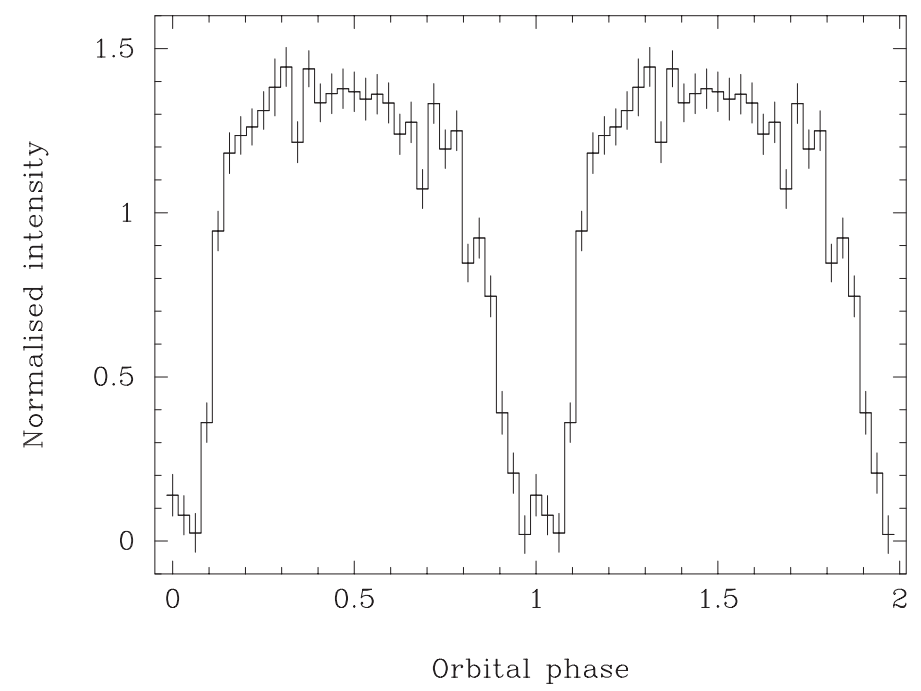

Figure 1. The RXTE-ASM light curve folded at the orbital period of $\sim 3.73$ days. Two orbital cycles are shown for clarity.

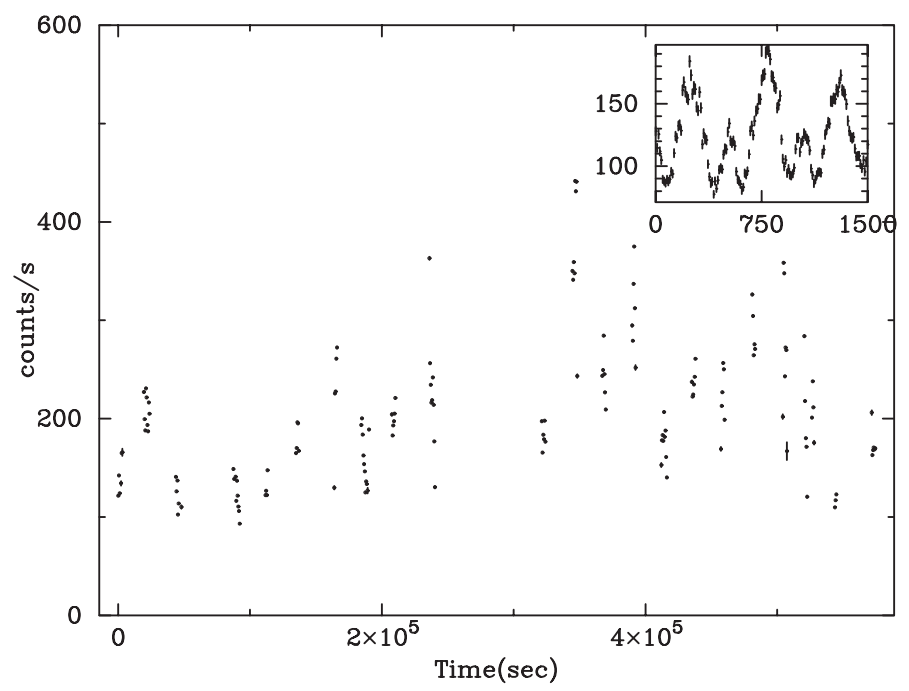

Figure 2. This plot shows the complete barycentered light curve for August 2003 data with a time bin of $526.85 \mathrm{~s}$, the same as the pulse period. The inset is an expanded view of a part of the total light curve with a time bin size of $10 \mathrm{~s}$. Individual double peaked pulses can be clearly seen.

be seen in the energy range from $1 \mathrm{keV}$ to $40 \mathrm{keV}$. The pulse profile is double peaked with a main pulse and an inter pulse which is about half the amplitude of the main pulse. The average pulse period of the source was obtained by pulse folding and $\chi^{2}$ maximisation method.

The light curve was then folded with the average spin period derived to get pulse profiles at different orbital phases. Each pulse profile is an average of two successive 


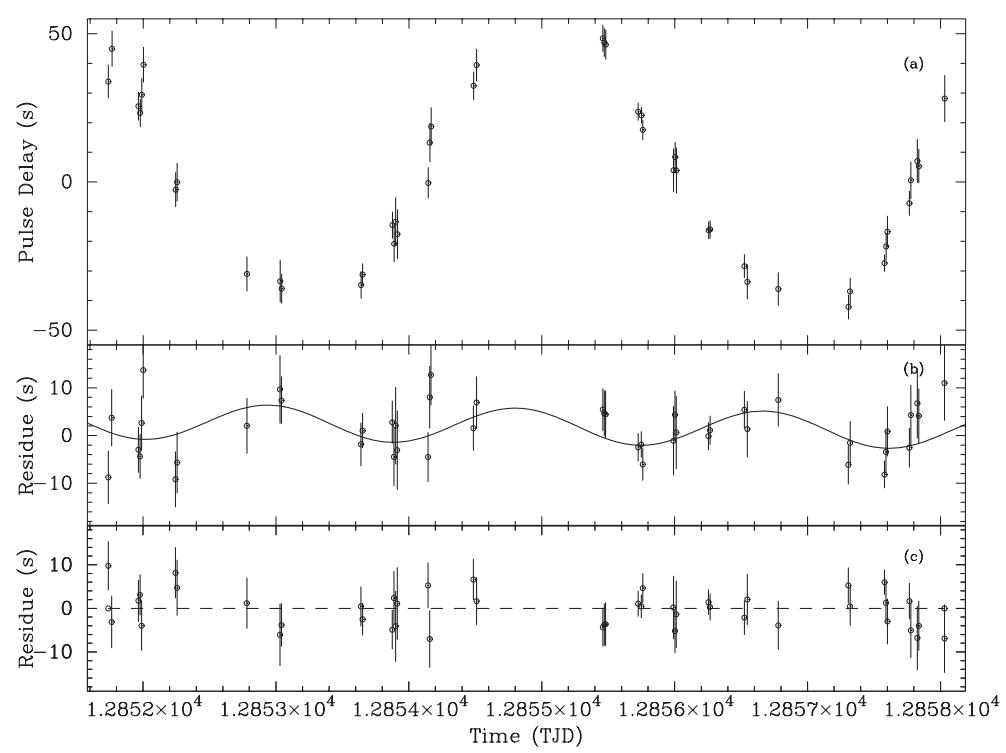

Figure 3. (a) Pulse arrival time delays measured from the RXTE-PCA observation in 2003, (b) residue of a circular fit to pulse arrival times, and (c) residue of an elliptical fit to pulse arrival times are shown here.

pulses binned into 256 pulse phase bins. A total of fortysix such pulse profiles were generated, spanned over two binary orbits of 4U 1538-52. To get the arrival times of these pulses, we decomposed each pulse profile into 128 Fourier components. The phase of the first Fourier component which also has the highest amplitude was used to find the arrival time delay of the respective pulses. Figure 3(a) shows the arrival time delay curve.

The arrival time delay curve when fitted with a circular orbital delay curve (i.e., $e=0$ ) leaves out systematic residues as shown in Fig. 3(b) indicating an elliptical orbit. Subsequently, we fitted the pulse arrival time delay with a function appropriate for elliptical orbit given below:

$$
\begin{aligned}
\Delta t & =\frac{a_{x} \sin i}{c}\left(1-e^{2}\right) \frac{\sin (v+\omega)}{1+e \cos v}, \\
\tan \frac{v}{2} & =\sqrt{\frac{1+e}{1-e}} \tan \frac{E}{2}, \\
E-e \sin E & =\frac{2 \pi}{P_{\text {orb }}}\left(t-T_{0}-\frac{P_{\text {orb }}}{2 \pi} \omega\right), \\
T_{0} & =T_{\pi / 2}-\frac{P_{\text {orb }}}{4} .
\end{aligned}
$$

It was seen that the errors in the individual measurements of pulse arrival times were greater than the error due to photon counting statistics, possibly due to some aperiodic intensity variation over a range of time scales. While fitting the arrival delay curve to an elliptical binary orbit, the statistical uncertainties on the arrival time measurements 
Table 1. The spin and orbital parameters of $4 \mathrm{U} 1538-52$ with $1 \sigma$ errors.

\begin{tabular}{ll}
\hline Parameter & \multicolumn{1}{c}{ Value } \\
\hline$P_{\text {spin }}$ & $526.849 \pm 0.003 \mathrm{~s}$ \\
$a_{x} \sin i$ & $53.1 \pm 1.5 \mathrm{lt}-\mathrm{s}$ \\
$\mathrm{e}$ & $0.18 \pm 0.01$ \\
$\omega$ & $40^{\circ} \pm 12$ \\
$T_{\pi / 2}$ & $52851.33 \pm 0.01 \mathrm{MJD}$ \\
$a_{o}$ & $47221.463 \pm 0.012 \mathrm{lt}-\mathrm{s}$ \\
$P_{\text {orb }}$ & $3.728382 \pm 0.000011 \mathrm{~d}$ \\
\hline$T_{\pi / 2}(N)=a_{0}+P_{\text {orb }} N$.
\end{tabular}

were multiplied by a constant factor to obtain a reduced $\chi^{2}$ value of 1.0. This method was employed so that one can compare the orbital parameter estimations with the earlier measurements (Clark 2000). For a circular orbit, the rescaled uncertainties gives a best fit with a $\chi^{2}$ value of 64 for 43 degrees of freedom. Comparing this with the reduced $\chi^{2}$ value of 1 for an elliptical orbit as described above, we reconfirm an elliptical orbit for this system. The value of the five free parameters for an elliptical fit, namely $P_{\text {spin }}, a_{x} \sin i$, e, $\omega$, and $T_{\pi / 2}$ are listed in Table 1 . We combined the new epoch measured from the RXTE data taken in 2003 with the earlier measurements listed in Clark (2000). The mid-eclipse times derived from light curves obtained using the UHURU and ARIAL V were not included because the X-ray eclipse is asymmetric as seen in the folded ASM light curve of Fig. 1. As the X-ray source goes into eclipse gradually and comes out of the eclipse rather sharply, the mid-eclipse time determined from the light curve only is likely to be inaccurate and have some systematic errors. Fitting the remaining 9 mid-eclipse times with a linear function gives a reduced $\chi^{2}$ of 1.3 for 7 degrees of freedom. The residuals from a linear fit to the mid-eclipse times are shown in Fig. 4. A quadratic fit improves the reduced $\chi^{2}$ by a mere 0.01 (for 6 degrees of freedom). If the mid-eclipse time record is fitted with an orbital evolution corresponding to the value reported in Clark (2000), a reduced $\chi^{2}$ of 2.5 is obtained, which is larger than the same for a linear fit. We, therefore, conclude that there is no evidence for an orbital evolution in 4U 1538-52 from the available data.

\section{Spectral analysis and results}

For the RXTE data, we took the Standard 2 data products of PCA and extracted the source spectra for each data set using the tool saextrct $v 4.2 \mathrm{~d}$ for the appropriate good time intervals. Background data files were generated with the tool runpcabackest using background models appropriate for the source brightness and the epoch of the RXTE observation, as provided by the PCA calibration team. The background subtracted source spectra were analyzed with the spectral analysis package XSPEC v 11.2.0 (Shafer et al. 1989). Model spectra were convolved with the detector response matrix and fitted to the observed pulse phase averaged spectrum in the spectral range of $3-20 \mathrm{keV}$ and the important spectral parameters were determined. The spectral model 


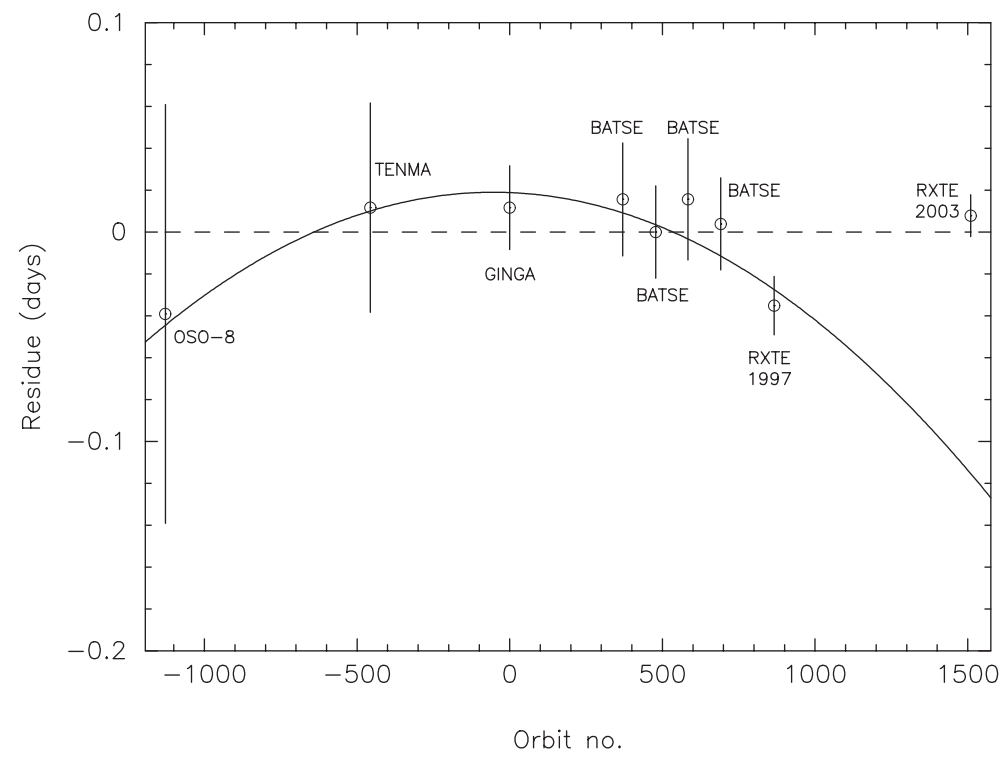

Figure 4. Differences between measured mid-eclipse times and a linear fit to the same are shown here. Solid line indicates the quadratic fit of mid-eclipse times till the 1997 observation (Clark 2000).

used was a powerlaw alongwith a high energy cut off and a Gaussian line with centre energy $\sim 6.4 \mathrm{keV}$ with a photoelectric absorption using Morrison and McCammon cross-sections (Morrison \& McCammon 1983). All the parameters were kept free in the 2003 data set while for the 1997 data set, the line centre energy and the width were kept frozen at $6.4 \mathrm{keV}$ and $0.01 \mathrm{keV}$ respectively.

The source and background spectra of BeppoSAX were extracted from the MECS and LECS detectors using circular regions of radius $4^{\prime}$ and $8^{\prime}$ respectively. The energy range chosen for MECS was (1.8-10.0) keV while that for LECS was $(0.3-4.5) \mathrm{keV}$, in which the respective instruments have large effective area and the spectral responses are well understood. For all the three observations, the MECS and LECS phase averaged out-of-eclipse spectra were fitted simultaneously with the relative normalization of the two instruments allowed to vary. All the spectra were suitably binned to allow use of $\chi^{2}$ statistics. Both the MECS and LECS data of BeppoSAX were simultaneously fitted using XSPEC v 11.2.0. Due to the restricted energy range $(1.8-10.0 \mathrm{keV})$, no high energy cutoff was required. The out of eclipse pulse phase averaged spectrum was first fitted with a simple model comprising of a powerlaw and a Gaussian line alongwith the photoelectric absorption using Morrison and McCammon cross-sections. The values of the line centre energy and line width as obtained in this fit were then kept frozen to fit spectra at different orbital phases.

The RXTE-PCA and the BeppoSAX spectra were all well fitted with a relatively simple model. Robba et al. (2001) had detected marginal evidence of a soft excess at $\sim 1 \mathrm{keV}$ which was resolved by modeling the spectrum with one additional component, either as a black-body of temperature $0.08 \mathrm{keV}$ or a bremsstrahlung of temperature $0.1 \mathrm{keV}$. We also found that the inclusion of a soft black-body component in the phase averaged out-of-eclipse spectrum from BeppoSAX marginally improves 


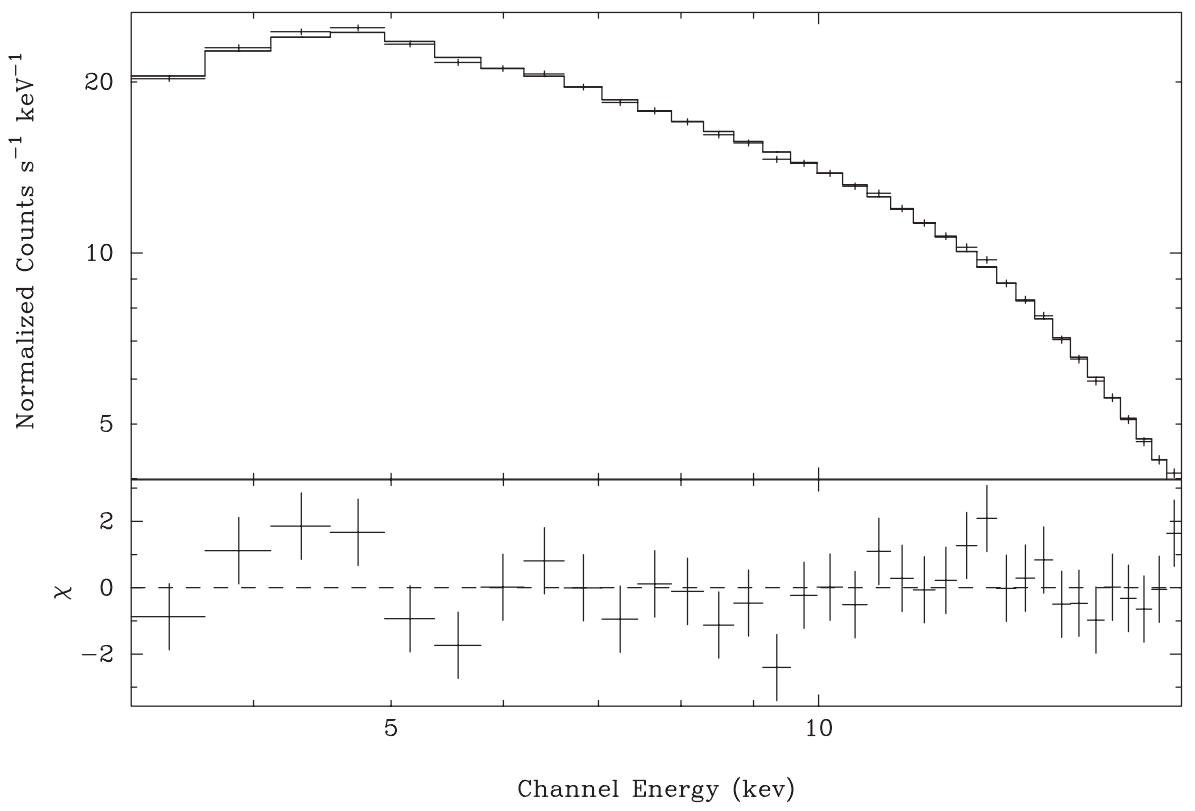

Figure 5. RXTE-PCA spectrum from an observation in 2003 corresponding to the orbital phase $\sim 0.28$. The residuals are shown in the lower panel. Soft excess was not included as it lies outside the energy range.

the fit. However, for orbital phase resolved spectroscopy, we have not included a soft component as it lies outside the range of the RXTE spectrum and in the orbital phase resolved spectrum with BeppoSAX, it will be too faint to constrain. In total, there were seventy nine spectra to be fitted and with the above-mentioned model, they gave reasonably reduced $\chi^{2}$ values in the range of 0.8 to 1.5 for RXTE (with 27 dof), and 0.6 to 1.3 for BeppoSAX (with 63 dof). Representative spectra, one each from the RXTEPCA and BeppoSAX are shown in Figs. 5 and 6 respectively. The RXTE-PCA spectra analysed here also does not include the energy range of the cyclotron absorption feature.

The spectral parameters obtained from the fitting of the three data sets used in our analysis were investigated for their variation against the orbital phase of the pulsar. The photon index of the power law is seen to remain more or less constant over the binary phase having values between 1.0 to 1.5 . The fluorescent iron-line flux measured with the average spectrum taken over 2000-3000 s, also does not show any considerable variation along the orbit. The cut-off energy measured with RXTE-PCA has a value $\sim 14 \mathrm{keV}$ and the e-folding energy is $\sim 7 \mathrm{keV}$. These are slightly different from the values of the cut-off energy $(\sim 16 \mathrm{keV})$ and e-folding energy $(\sim 10 \mathrm{keV})$ measured with BeppoSAX in a broad energy band (Robba et al. 2001). We detected a notable variation in the equivalent hydrogen column density with orbital phase as shown in Fig. 7. It shows a smooth variation over orbital phase, increasing gradually by an order of magnitude as the pulsar approaches eclipse (mid-eclipse is defined by phase zero). At orbital phases far from the eclipse, the column density has a value of $\sim 1.5 \times 10^{22} \mathrm{H}$ atoms $\mathrm{cm}^{-2}$. In Figs. 1, 5, 6, and 7, the orbital phase is measured with respect to the mid-eclipse time. 


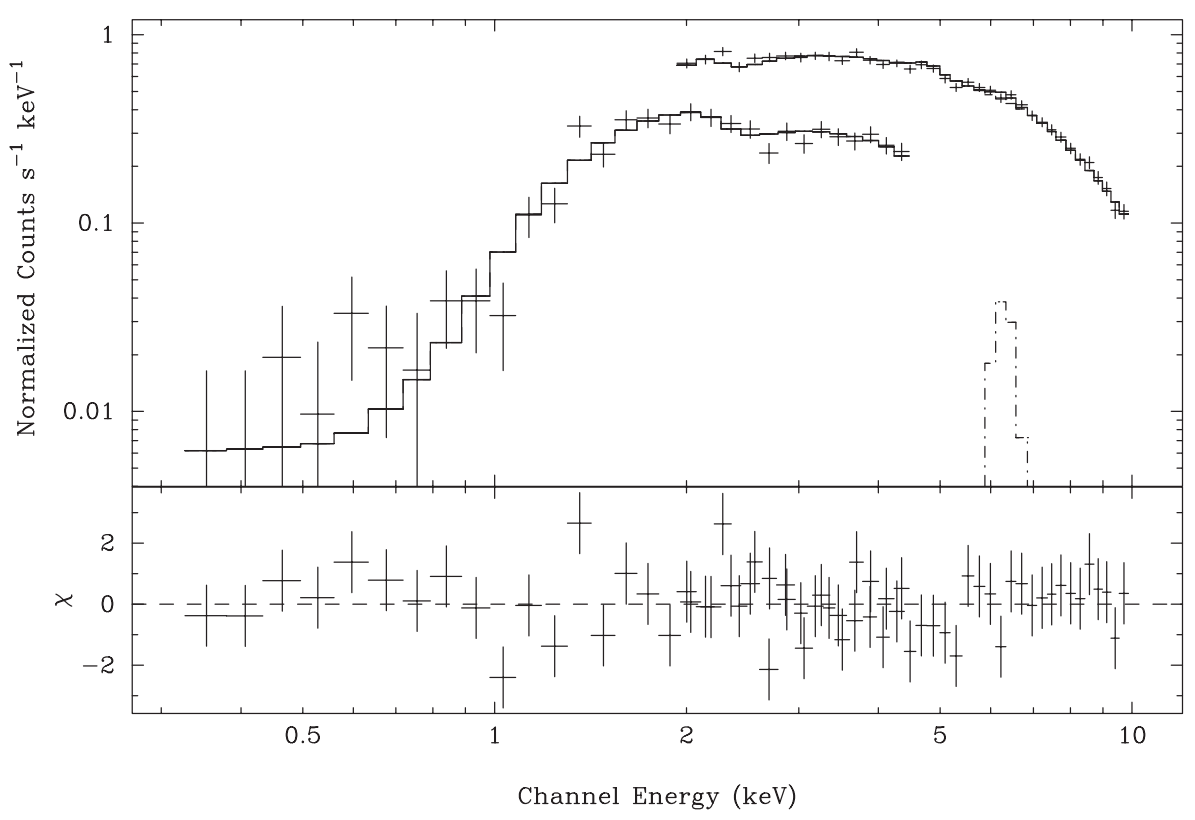

Figure 6. A BeppoSAX spectrum corresponding to orbital phase $\sim 0.59$ with the residuals in the lower panel. The narrow Gaussian component at $6.4 \mathrm{keV}$ shown here is the iron fluorescence line.

\section{Discussion}

A pulse period of $526.85 \mathrm{~s}$ of the pulsar $4 \mathrm{U}$ 1538-52 determined from RXTE-PCA observation in 2003 shows that the spin-up trend of 4U 1538-52 first detected with BATSE during the 1990 s has continued since then. The overall spin-up time scale $(\dot{P} / P)$ during the five years of BATSE observation reported by Rubin et al. (1997) was $9.6 \times 10^{-12} \mathrm{~s}^{-1}$. The average spin-up rate between the end of BATSE observations and the later observations with RXTE (1997, Clark 2000), Beppo-SAX (1998, Robba et al. 2001) and again with RXTE (2003, present work) is $1.4,1.2$ and $7.4 \times 10^{-11} \mathrm{~s}^{-1}$, significantly larger than the average spin-up rate during the BATSE era. An increase in spin-up rate is expected if the overall X-ray luminosity had increased after the BATSE observations. However, it is very impractical to compare the pulsed hard X-ray flux measured by BATSE and the total X-ray flux measurements with the later instruments in a different energy band.

The value of $a_{x} \sin i$ that we obtained from pulse timing of the RXTE-PCA data in 2003 is $53.1 \pm 1.5 \mathrm{lt}-\mathrm{s}$, which is consistent with the BATSE result and slightly smaller than that obtained from the 1997 RXTE data. We note here that the accuracy of orbital parameter determination in slow pulsars like $4 \mathrm{U} 1538-52$ is considerably poorer than in fast pulsars like SMC X-1, Her X-1, etc. In the analysis of BATSE data, Rubin et al. (1997) considered a circular orbit whereas analysing pulse arrival times from two complete binary orbits we found the orbit of 4U 1538-52 to be eccentric with $e=$ $0.18 \pm 0.01$. Our result is consistent with the results obtained from a 1997 observation of this source with the RXTE-PCA (Clark 2000). The value of $\omega$ determined from the 2003 observation is smaller compared to that found by Clark from the 1997 observation. 


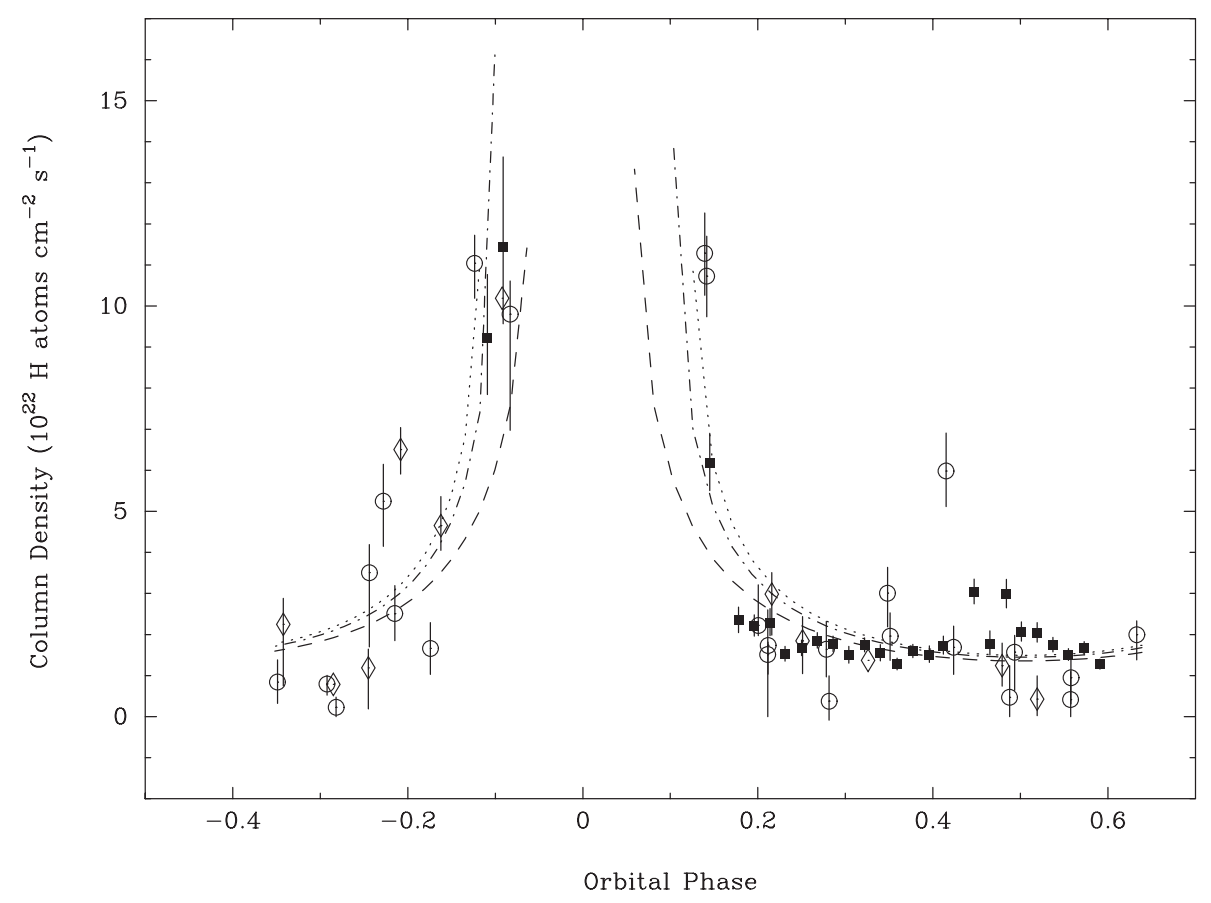

Figure 7. Variation of column density versus orbital phase with respect to the mid-eclipse time is shown here. The dashed line represents the model for inclination angle of $65^{\circ}$, the dashed-dotted line for inclination angle of $75^{\circ}$ and the dotted line for inclination angle $85^{\circ}$. Diamonds, filled squares, and circles denote measurements from observations made with RXTE in 1997, BeppoSAX in 1998, and RXTE in 2003 respectively. The error bars shown correspond to a $90 \%$ confidence interval.

If the difference is due to apsidal motion then the rate of apsidal motion is $\dot{\omega}=$ $-3.8^{\circ} \pm 2^{\circ} .2 \mathrm{yr}^{-1}$. We found no significant evidence for orbital evolution in the system as reported from earlier measurements (Clark 2000). The value of $\dot{P}_{\text {orb }} / P_{\text {orb }}$ determined from all the available mid-eclipse times is $(2.5 \pm 1.9) \times 10^{-6} \mathrm{yr}^{-1}$. Combining the pulse timing results on $4 \mathrm{U} 1538-52$ obtained from our observation with the earlier reported measurements, we are able to rule out an orbital decay in this system for which Clark (2000) found marginal evidence. The new upper limit presented here on possible changes of the orbital period of 4U 1538-52 supersedes previous limits considerably. ${ }^{1}$

Tidal interaction between the two stars and mass loss from the companion star are the two main effects that cause orbital decay in the HMXB systems. The orbital evolution of several HMXB pulsar systems (Cen X-3, SMC X-1 and LMC X-4) is very well documented. The companion star mass, and the size of the binary orbit in $4 \mathrm{U} 1538$ 52 is very similar to the above-mentioned binaries, and therefore, the tidal effect that is expected in $4 \mathrm{U}$ 1538-52 should be as strong as in the three other HMXBs. In the present work, we find no clear evidence of an orbital decay in 4U 1538-52. If at all, the system shows a positive orbital period derivative, like the enigmatic system Cyg

\footnotetext{
${ }^{1}$ After this paper was submitted, we came to know about a paper by Baykal, Inam and Beklen (2006). These authors have used a different analysis method and reached a similar conclusion about the orbital period evolution of $4 \mathrm{U} 1538-52$.
} 
X-3 (Singh et al. 2002). This indicates that mass loss may play a prominent role in the evolution of this system. Tidal interaction between the neutron star and the companion in close HMXBs should also circularise the orbit. The orbits of most of the close (orbital period less than 4 days) HMXBs are highly circularised with eccentricity less than 0.006 . But the orbit of $4 \mathrm{U} 1538-52$ is not yet circularised and shows no orbital decay. Therefore, it is likely that $4 \mathrm{U} 1538-52$ is a relatively young system compared to the other HMXBs. Using the mass, radius and luminosity of the companion star QV Nor from the optical observation (Reynolds et al. 1992) the approximate time for tidal circularisation of this system works out to be $2 \times 10^{3} \mathrm{yr}$ (Lecar et al. 1976). Since the system is still eccentric, it is possible that the age of the system after the supernova explosion is of the order of a few thousand years; very small compared to the lifetime of HMXBs.

The eccentricity derived using the two RXTE observations in 1997 (Clark 2000) and 2003 (reported here) is likely to be correct for two reasons. The RXTE-PCA has a large effective area compared to the earlier missions and in the energy range of PCA more photons are detected compared to CGRO-BATSE using which Rubin et al. (1997) did not detect any eccentricity. If the eccentricity is high, then either the binary system is young or the circularisation time is actually much longer than a few thousand years estimated using Lecar's method (1976). On the other hand, a binary is unlikely to become very X-ray active by wind accretion within just a few thousand years of the supernova explosion. It is possible that the circularisation mechanism works very efficiently in systems with Roche-lobe overflow like LMC X-4 while accretion mechanism in 4U 1538-52 is likely to be driven by stellar wind.

Lack of any substantial variation in the photon index, the cut-off energy, and the e-folding energy over the binary phase of 4U 1538-52 suggests that the continuum $\mathrm{X}$-ray spectrum of the pulsar is hardly affected during its revolution along the orbit. This indicates a nearly constant accretion rate and identical conditions in the accretion column throughout the binary orbit. In the HMXB pulsar GX 301-2, orbital phase resolved spectroscopy with RXTE-PCA showed that the spectral parameters remain almost constant throughout the orbit though the overall accretion rate and wind density, and clumpiness of the wind changes considerably with the binary phase (Mukherjee \& Paul 2004). On the other hand, in 4U 1538-52 the equivalent hydrogen column density shows a smooth variation over the orbital phase. We compare the observed column density profile with a model estimated by assuming a spherically symmetric Castor et al. (1975) wind from the companion star. The velocity profile of the wind is:

$$
v_{\text {wind }}=v_{\infty} \sqrt{1-\frac{R_{\mathrm{c}}}{r}}
$$

where $v_{\infty}$ is the terminal velocity for the stellar wind, $R_{c}$ is the radius of the companion and $r$ is the radial distance from centre of the companion star. The column density profiles were derived using a numerical integration along the line of sight from the pulsar to the observer for three different inclination angles $65^{\circ}, 75^{\circ}$ and $85^{\circ}$ respectively. In Fig. 7, we show the equivalent hydrogen column density measured with BeppoSAX and RXTE-PCA as a function of the orbital phase with respect to the mid-eclipse time. A mass-loss rate of $\sim 10^{-6} \mathrm{M}_{\odot} \mathrm{yr}^{-1}$ and $v_{\infty} \sim 1000 \mathrm{~km} \mathrm{~s}^{-1}$ were assumed. The model calculations of the absorption column density for different inclination angles when superposed on the observed values indicate reasonable agreement as can be seen in Fig. 7. This indicates that a spherically symmetric stellar wind from the companion 
star may produce the observed orbital dependence of the column density for certain range of the orbital inclination.

We note here that we have not done any fitting of the column density measurements and the model column density values at different orbital phases (Fig. 7). For the wind density model used here, the phase resolved column density values depend on the mass loss rate, the terminal velocity and the inclination angle of the binary orbit. The data presented here is not suitable for such a detailed analysis but only shows that such a model is consistent with the observations. In eclipsing X-ray binaries like 4U 1538-52, the eclipse duration puts strong constraints on the orbital inclination. We point out that the orbital phase resolved column density measurements can be an independent way of estimating the orbital inclination, especially for non-eclipsing X-ray binaries. We also note that in several eclipsing and wind-fed HMXBs, the eclipse ingress is more gradual than the eclipse egress (Haberl et al. 1989; Feldmeier et al. 1996; Naik \& Paul 2004). From the RXTE-ASM light curves of many eclipsing HMXB pulsars we have verified that this effect is more pronounced at lower energies. This ingress-egress asymmetry, which varies in degree from source to source is partly due to absorption by accretion material trailing the X-ray source in its orbit. For example, Vela X-1 (Feldmeier et al. 1996) has a much larger ingress-egress asymmetry and LMC X-4 (Naik \& Paul 2004) has a much smaller asymmetry compared to $4 \mathrm{U}$ 1538-52 (Fig. 1). We think that the slight asymmetric accretion column distribution in the case of 4U 1538-52 on two sides of the eclipse (Fig. 7) can also partly be due to some trailing accreting material.

In several other HMXBs like 4U 1700-37 (Haberl et al. 1989), 4U $1907+09$ (Roberts et al. 2001) and GX 301-2 (Leahy 1991; Pravdo \& Ghosh 2001, with RXTE), it has been shown that a simple spherical wind emanating from the companion star is not sufficient to explain the column density profile. Density enhancement due to a trailing stream or a disk from the companion arising due to the dynamical effects of the pulsar was invoked to explain the said profile. In the case of GX 301-2, detailed observations with RXTE-PCA revealed that neither a disk nor a stream was sufficient to explain the observed profile (Mukherjee \& Paul 2004). Considering the results for these HMXBs, the present work seems to carry an interesting implication. Clark et al. (1994) have studied the column density variation during eclipse egress for this particular source with GINGA data. Two HMXB pulsars in which similar increase in absorption column density pattern was seen near eclipse are X1908+075 (Levine et al. 2004) and SMC X-1 (Woo et al. 1995) which also may have isotropic wind pattern from the companion stars. In the present study we investigated the out-of-eclipse variation of the column density and found that a simple model with a spherically symmetric stellar wind describes the observations well. Since $4 \mathrm{U} 1538-52$ has a moderate X-ray luminosity $\left(\sim 10^{36} \mathrm{erg} \mathrm{s}^{-1}\right)$, it is not expected to cause any significant perturbation in the acceleration of the stellar wind through X-ray ionization as compared to high luminosity sources like Cen X-3 (Day \& Stevens 1993) and SMC X-1 (Woo et al. 1995) with luminosities of $\sim 10^{38} \mathrm{erg} \mathrm{s}^{-1}$ in the high states. Observation of $4 \mathrm{U} 1538$ 52 covering the eclipse ingress and egress with high throughput telescope like XMMNewton and SUZAKU which also have good low energy response will allow one to carry out a detailed study of the stellar wind structure in this source and also determine the extent to which the local X-ray photoionisation affects the stellar wind acceleration. 


\section{Acknowledgements}

We thank an anonymous referee for some useful suggestions that helped us to improve the paper. This research has made use of data obtained from the High Energy Astrophysics Science Archive Research Center (HEASARC), provided by NASA's Goddard Space Flight Center. We also thank the BeppoSAX team for making the data available. UM and HR would like to acknowledge the Kanwal Rekhi Scholarship of TIFR Endowment Fund for partial financial support.

\section{References}

Boella et al. 1997, A\& AS, 122, 299.

Castor, J. I., Abbott, D. C., Klein, R. I. 1975, ApJ, 195, 157.

Clark, G. W., Woo, J. W., Nagase, F., Makishima, K., Sakao, T. 1990, ApJ, 353, 274.

Clark, G. W., Woo, J. W., Nagase, F. 1994, ApJ, 422, 336.

Clark, G. W. 2000, ApJ, 542, 131L.

Davison, P. J. N., Watson, M. G., Pye, J. P. 1977, MNRAS, 181, 73.

Day, C. S. R., Stevens, I. R. 1993, ApJ, 403, 322.

Feldmeier, A., Anzer, U., Boerner, G., Nagase, F. 1996, A \& A, 311, 793.

Giacconi et al. 1974, ApJS, 27, 37.

Haberl, F., White, N. E., Kallman, T. R. 1989, ApJ, 343, 409.

Jahoda et al. 1996, In: EUV, X-Ray and Gamma-Ray Instrumentation for Astronomy VII (eds.) Siegmund, O. H. W., Gummin, M. A., SPIE 2808, p. 59.

Leahy, D. A. 1991, MNRAS, 250, 310L.

Lecar, M., Wheeler, J. C., McKee, C. F. 1976, ApJ, 205, 556L.

Levine, A. M., Rappaport, S. A., Zojcheski, G. 2000, ApJ, 541, 194L.

Levine, A. M., Rappaport, S., Remillard, R., Savcheva, A. 2004, ApJ, 617, 1284.

Makishima, K., Koyama, K., Hayakawa, S., Nagase, F. 1987, ApJ, 314, 619.

Morrison, McCammon 1983, ApJ, 270, 119.

Mukherjee, U., Paul, B. 2004, A \& A, 427, 567.

Nagase, F., Corbet, R. H. D., Day, C. S. R., Inoue, H., Takeshima, T., Yoshida, K., Mihara, T. 1992, ApJ, 396, 147.

Naik, S., Paul, B. 2004, ApJ, 600, 351.

Parkes, G. E., Murdin, P. G., Mason, K. O. 1978, MNRAS, 184, 73.

Pravdo, S. H., Ghosh, P. 2001, ApJ, 554, 383.

Reynolds, S. A., Bell, S. A., Hilditch, R. W. 1992, MNRAS, 256, 631.

Robba, N. R., Cusumano, G., Orlandini, M., dal Fiume, D., Frontera, F. 1992, ApJ, 401, 685.

Robba, N. R., Burderi, L., Di Salvo, T., Iaria, R., Cusumano, G. 2001, ApJ, 562, 950.

Roberts et al. 2001, ApJ, 555, 967.

Rubin, B. C., Finger, M. H., Scott, D. M., Wilson, R. B. 1997, ApJ, 488, 413.

Shafer, R. A., Haberl, F., Arnaud, K. A. 1989, XSPEC: An X-ray Spectral Fitting Package, ESA TM-09 (Paris: ESA).

Singh, N. S., Naik, S., Paul, B., Agrawal, P. C., Rao, A. R., Singh, K. Y. 2002, A \& A, 392, 161. Wojdowski, P., Clark, G. W., Levine, A. M., Woo, J. W., Zhang, S. N. 1998, ApJ, 502, 253. Woo, J. W., Clark, G. W., Blondin, J. M., Kallman, T. R., Nagase, F. 1995, ApJ, 445, 896. 